\title{
Cobertura da Estratégia Saúde da Família e de citopatologia de colo uterino no Rio Grande do Sul
}

\author{
Coverage of the Family Health Strategy and uterine \\ colon citopathology in Rio Grande do Sul
}

Resumo

Paulo Vinícius Nascimento Fontanive ${ }^{1}$ João Henrique Godinho Kolling ${ }^{2}$ Eno Dias de Castro Filho ${ }^{3}$ Erno Harzheim ${ }^{4}$

O Rio Grande do Sul apresentou nos últimos cinco anos aumento significativo na cobertura populacional da Estratégia Saúde da Família (ESF). A mudança na organização da Atenção Primária à Saúde (APS) proposto pela ESF já demonstrou, em grandes centros, melhora no acesso e em outros atributos da APS, conforme estudos que avaliaram indicadores de saúde infantil ao comparar a ESF com a Atenção Básica Tradicional. Buscou-se analisar a associação entre a cobertura populacional da ESF com a cobertura de citopatológico de colo uterino entre mulheres de 25 a 59 anos nos municípios do Rio Grande do Sul. Este é um estudo ecológico que usou base de dados secundários do DATASUS oriundos do SISPACTO no período de janeiro a dezembro de 2006. Para avaliar o acesso a ações em saúde da mulher foi escolhido um dos indicadores do pacto pela saúde, a razão de exames citopatológicos, que diz respeito ao número de exames citopatológicos cérvico-vaginais (CP) realizados na população de interesse. Os dados foram estratificados em seis grupos conforme cobertura populacional dos municípios (sem ESF, até $20 \%$, de $20 \%$ a $40 \%$, de $40 \%$ a $60 \%$, de $60 \%$ a $80 \%$ e mais de $80 \%$ de cobertura). Para avaliar a influência do porte populacional, os municípios com menos de 10 mil foram comparados com os demais. Na análise utilizou-se o programa SPSS v13.0, realizando a análise de variância e teste post hoc (Tukey) para comparação entre os grupos de cobertura e teste $\mathrm{T}$ para comparação de médias conforme o porte populacional. Como resultado, um grupo de maior cobertura apresentou diferença estatisticamente significativa com os grupos de cobertura menor que $60 \%(p<0,05)$. Nota-se que nos grupos de cobertura há diferença estatisticamente significativa entre as razões de CP de acordo com o porte populacional. Quando apenas analisamos os municípios com mais de 10 mil habitantes, observamos que os municípios com mais de $60 \%$ de cobertura apresentaram uma razão de exames de CP estatisticamente maior que aqueles com até $20 \%$ ( $p<0,01)$. De acordo com os achados percebe-se que mudanças no modelo de organização do cuidado por meio da maior cobertura municipal da ESF também se associam com melhora em indicadores de ações em saúde da mulher.

Palavras-chave: Atenção Primária à Saúde;

Saúde da Mulher; Programa Saúde da Familia;

Esfregaço Cervical; Neoplasias Uterinas.

\author{
Key Words: Primary Health Care; \\ Women's Health; Family Health Program; \\ Vaginal Smears; Uterine Neoplasms.
}

${ }^{1}$ Cirurgião-dentista, especialista em Saúde Pública, mestrando em Epidemiologia pela PPGEPI - UFRGS. Especialista em Saúde Pública, Porto Alegre, Rio Grande do Sul, Brasil.

${ }^{2}$ Médico de Família e Comunidade, Especialista em Medicina de Família e Comunidade, mestrando em Epidemiologia pela PPGEPI - UFRGS, Porto Alegre, Rio Grande do Sul, Brasil.

${ }^{3}$ Mestre em Educação Médico de Família e Comunidade, mestre em Educação, doutorando em Epidemiologia pela PPGEPI - UFRGS, Serviço de Saúde Comunitária, Grupo Hospitalar Conceição, Porto Alegre, Rio Grande do Sul, Brasil.

${ }^{4}$ Médico de Família e Comunidade, doutor em Saúde Pública pela Universidade de Alicante (Espanha), coordenador Grupo de Pesquisa em APS de Porto Alegre, Departamento de Medicina Social, Universidade Federal do Rio Grande do Sul, Porto Alegre, Rio Grande do Sul, Brasil 


\section{Abstract}

Rio Grande do Sul showed in the last five years a significant increase in the population coverage provided by the Family Health Strategy (FHS). The changes in the organization of Primary Care proposed by the FHS already demonstrated in the great urban centers improvement in the access and other attributes of Primary Care services, as demonstrated in studies that evaluated indicators of child health when comparing the FHS with the traditional Primary Care services. This study sought analyzing the association between the population coverage of the FHS and the coverage of citopathological exams of the uterine colon among women between 25 and 59 years of age in cities of Rio Grande do Sul. This is an ecological study that used the base of secondary data of the DATASUS originating from SISPACTO over the period January to December 2006. One of the indicators of the health pact was chosen for evaluating the actions directed to women's health - the number of cervicovaginal citopathological exams performed in the population of interest. The data were stratified into 6 groups according to the population coverage in the cities (without FHS, up to 20\%, from 20\% to 40\%, from $40 \%$ to $60 \%$, from $60 \%$ to $80 \%$ and more than $80 \%$ of coverage). For evaluating the influence of the population size, cities with less than 10 thousand inhabitants were compared with the others. The analysis was performed using the software SPSS 13.0 for variance analysis and post hoc test (Turkey) for comparison between the coverage groups and $T$ test for comparison of means according to the population size. As a result, one group of greater coverage showed a statistically significant difference from the group with less than $60 \%$ of coverage $(p<0,05)$. It has to be noted that in the covered groups there is a statistically significant difference between the ratios of citopathological exams (CP) according to the population size. Analyzing only the cities with more than 10 thousand inhabitants, we observe that cities with more than $60 \%$ of coverage presented a ratio of CP exams statistically higher than those wit up to 20\% ( $p<0,01)$. These findings show that changes in the organizational model of care through a greater FHS coverage in the cities are also associated with an improvement of the indicators of women's health actions.

\section{Introdução}

A organização de uma rede hierarquizada, descentralizada, regulada por meio de instâncias de controle social e balizada por princípios como eqüidade, integralidade e universalidade de acesso se constitui em um desafio permanente desde a criação do Sistema Único de Saúde (SUS). A reorganização do modelo assistencial é um passo imprescindível para o fortalecimento de um sistema público de saúde viável e efetivo. Os sistemas de saúde nacionais que apresentaram melhores resultados sobre a situação de saúde de sua população fundamentam esta organização sobre uma bem estruturada rede de Atenção Primária à Saúde $^{1,2}$. A Estratégia Saúde da Família (ESF) surge como uma proposta de caráter substitutivo que resgata os atributos da APS propostos por Starfield ${ }^{3}$ : acesso (primeiro contato), integralidade, longitudinalidade e coordenação do cuidado, além de abordagem comunitária, familiar e competência cultural.

A ESF no Rio Grande do Sul apresentou grande incremento da cobertura nos últimos anos, da mesma forma que no restante do Brasil, porém desenvolvendo-se de forma mais tardia. Entre dezembro de 2000 e de 2007, a cobertura populacional da ESF saltou de 6,8\% para 31,8\%, neste estado, passando de cerca de 200 equipes para mais de 1.060 no mesmo período. Em dezembro de 2007, 390 municípios já contavam com equipes da ESF na rede de Atenção Primária à Saúde (APS). ${ }^{4}$

Este aumento significativo permite uma mudança quanto ao acesso a serviços de saúde e principalmente quanto à qualidade do cuidado e seu possível impacto sobre a saúde da população. Resultados da expansão da ESF no Brasil foram relatados em estudo ecológico, no qual se observou que o aumento de $10 \%$ na cobertura da ESF se associava à diminuição de 4,5\% no índice de mortalidade infantil, mesmo corrigindo para os determinantes clássicos desse indicador ${ }^{5}$. No nível individual, estudo de base populacional sobre a APS oferecida a crianças, cuja qualidade foi referida pelos cuidadores, comparou a ESF e as Unidades Básicas de Saúde Tradicionais (UBS) em Porto Alegre. Foi demonstrada maior adscrição ao serviço de crianças 
residentes nas áreas da ESF, e melhor qualidade do processo de atenção como vigilância do crescimento, prescrição mais freqüente de vitamina $\mathrm{D}$ e sulfato ferroso em lactentes e maior satisfação com o serviço de saúde ${ }^{6,7}$. Estudos em adultos demonstram que os serviços da ESF possuem maiores escores dos atributos essenciais de APS em relação às Unidades Básicas de Saúde Tradicionais, medidos pelo instrumento validado PCATool ${ }^{8,9}$.

O Departamento de Atenção Básica (DAB) do Ministério da Saúde (MS) elaborou recentemente uma relação dos agravos que são considerados condições sensíveis ao escopo de ações da Atenção Primária à Saúde. Entre os agravos nesta relação, encontram-se as neoplasias de colo de útero e outras condições que tangem à saúde da mulher. Simultaneamente, o Pacto pela Saúde (Portaria 325 de 2008) ${ }^{10}$ priorizou o acompanhamento e a pactuação deste agravo por meio do indicador de cobertura populacional de exames preventivos do câncer de colo uterino. Dessa forma, a APS figura como peça central na prevenção e na redução das estatísticas de morbimortalidade deste problema de saúde pública.

O câncer de colo de útero é uma das principais causas de morte entre as mulheres no Brasil e no Rio Grande do Sul. Segundo estimativas do Instituto Nacional de Câncer (INCA), o câncer de colo uterino é o segundo mais incidente na região Sul. Esta região é a que apresenta maior estimativa de novos casos para o ano 2008 no país: 24 por 100 mil mulheres. No Rio Grande do Sul a taxa de incidência é de 29,5 por 100 mil mulheres ${ }^{11}$. A taxa de mortalidade no Estado do Rio Grande do Sul para o câncer de colo foi de 11,6 óbitos por 100 mil mulheres acima de 30 anos no ano de 2005, um pouco superior à média da região Sul, que foi de 11,1 óbitos $^{12}$.

Mas, afinal, o quanto este problema é vulnerável às ações de um Sistema de Saúde? Embora nunca tenham sido realizados ensaios clínicos randomizados avaliando o rastreamento do câncer de colo uterino, grandes estudos observacionais estimam uma redução de $60 \%$ a $80 \%$ da incidência por este câncer três anos após a implementação de um programa de rastreamento de mulheres com o teste de Papanicolau (citopatologia esfoliativa ou CP) e o encaminhamento para tratamento das lesões precursoras com alto potencial de malignidade ou carcinoma in situ ${ }^{13,14}$. Há correlação entre rastreamento e mortalidade, demonstrado em estudos europeus e norte-americanos, com redução nas curvas de mortalidade entre $20 \%$ a $40 \%{ }^{15}$ nas populações rastreadas. Este conjunto de ações está elencado entre as responsabilidades da Atenção Primária à Saúde no Brasil quanto à sua execução e quanto ao seu acompanhamento e monitoramento.

O Ministério da Saúde, em sua publicação normativa para a APS sobre a linha de cuidados na prevenção e diagnóstico precoce do câncer de colo uterino, recomenda a realização do exame dos 25 aos 60 anos, uma vez por ano, e, após dois exames negativos, a cada três anos ${ }^{16}$. Esta recomendação representa uma razão de 0,33 exames citopatológicos de colo uterino entre mulheres de 25 a 59 anos ao ano, meta sugerida pelo SISPACTO. Nesse mesmo documento, o diagnóstico tardio do câncer cérvico-vaginal é relacionado à dificuldade de acesso aos serviços e à incapacidade destes absorverem a demanda que chega às unidades de saúde, entre outras razões.

Revisão sistemática sobre estudos avaliando a cobertura de realização do CP no Brasil observou que os principais fatores relacionados à não-realização do mesmo são baixo nível socioeconômico, baixa escolaridade, baixa renda familiar e faixas etárias mais jovens ${ }^{17}$. No entanto, o autor da revisão relata que todos os inquéritos identificados tiveram foco individual, de cunho sociodemográfico e comportamental, e não conseguiram avaliar associação da organização dos serviços sobre o acesso ao exame. Este estudo tem por objetivo identificar a associação de uma maior cobertura populacional da ESF com a cobertura de citopatológico de colo uterino em mulheres de 25 a 59 anos nos municípios do Rio Grande do Sul.

\section{Metodologia}

População, bases de dados e variáveis

Faz parte deste estudo o universo dos municípios do estado do Rio Grande do Sul (n=496). Este estudo eco- 
lógico avaliou a cobertura populacional da ESF obtida por meio da divisão da população municipal coberta pelos serviços da ESF, segundo dados do DAB, pela população total do município, conforme estimativa IBGE do ano de 2006. Vale ressaltar que o DAB define cobertura a partir de uma constante de 3.450 habitantes por equipe. Assim, as coberturas populacionais podem superar $100 \%$ onde as equipes atendem populações menores.

Para avaliar as ações de prevenção do câncer de colo de útero foi escolhido um dos indicadores do Pacto pela Saúde, a razão de exames citopatológicos. Este indicador é definido pela razão entre o número de exames citopatológicos cérvico-vaginais (CP) realizados em mulheres de 25 a 59 anos durante o ano de 2006 e a população feminina nessa faixa etária no mesmo período.

Trata-se de um estudo que utilizou base de dados secundárias disponíveis por meio do TABNET, instrumento de busca de informações do Departamento de Informática do SUS (DATASUS). Os dados extraídos provinham do Sistema de Informações da Atenção Básica (SIAB), Sistema de Informações Ambulatoriais (SIA), de informações do Instituto Brasileiro de Geografia e Estatística (IBGE), e estavam agrupados no Sistema de Informações do Pacto (SISPACTO).

\section{Estratificação por grupos de cobertura}

Os municípios foram estratificados em seis grupos conforme cobertura populacional pela Estratégia Saúde da Família descritos no quadro 1.
A separação dos grupos 1 e 2, respectivamente, sem ESF e com cobertura de ESF entre 0,01 a $20 \%$ foi intencional, em função de características intrínsecas deste grupo de municípios. De maneira geral, os municípios com coberturas entre $0,01 \%$ e $20 \%$ incluem a maioria dos municípios com mais de 100 mil habitantes (participantes do Programa de Expansão do Saúde da Família - PROESF). Já entre os 91 municípios sem ESF apenas dois municípios possuem mais de 100 mil.

Para avaliar a influência do porte populacional, a análise foi estratificada entre os municípios com até 10 mil e mais de 10 mil habitantes. Neste último grupo, diante da redução no número de municípios por grupo, em especial nos grupos de $0,01 \%$ a $20 \%$ e de $20,01 \%$ a $40 \%$, optamos pelo agrupamento dos mesmos em novo grupo de $0,01 \%$ a $40 \%$ de cobertura.

\section{Análise estatística}

Foi utilizado o programa SPSS v13.0, importando os dados salvos em formato DBF4 do programa TABWIN (disponível pelo DATASUS). Realizamos a análise de variância (one way ANOVA) para verificar diferenças estatisticamente significativas da razão de CP entre os diversos estratos de cobertura populacional da ESF, com o uso do teste de Tukey como post hoc.

Para identificar se os grupos de municípios com menos de 10 mil e mais de 10 mil apresentam diferença estatisticamente significativa na razão de exames preventivos foi realizado teste $T$ para comparação de médias entre as

Quadro 1. Distribuição dos municípios do RS entre os grupos de cobertura populacional da ESF e o número de municípios para cada grupo:

\begin{tabular}{ccc}
\hline Grupos & Cobertura populacional & $\mathbf{N}^{\text {o }}$ de municípios \\
\hline$(1)$ & sem ESF & 91 \\
$(2)$ & entre 0,01 e $20 \%$ de ESF & 44 \\
$(3)$ & entre $20,01 \%$ e $40 \%$ de ESF & 51 \\
$(4)$ & entre $40,01 \%$ e $60 \%$ de ESF & 43 \\
$(5)$ & entre $60,01 \%$ e $80 \%$ de ESF & 41 \\
$(6)$ & Mais de $80,01 \%$ de ESF & 225 \\
Total & & 496 \\
\hline
\end{tabular}

122 Rev Bras Med Fam e Com

Rio de Janeiro, v.4, $n^{\circ} 14$, jul/set 2008 
duas categorias de porte populacional.

Após a verificação da diferença entre as médias destes dois grupos de municípios por meio do teste $\mathrm{T}$ foi realizada nova análise de variância (one way ANOVA) da razão de CP entre os diversos estratos de cobertura populacional da ESF, com o uso do teste de Tukey como post hoc, de acordo com o porte populacional (municípios com mais ou menos de 10 mil habitantes). Em todas as análises, foi considerado nível de significância (alfa) de 0,05 bicaudal.

Para verificar o grau de associação e de determinação da cobertura populacional da ESF e da razão de exames de CP, foi realizada regressão linear múltipla, controlandose para o porte populacional de todos os municípios (variável contínua). A entrada e a saída das variáveis do modelo ocorreram de acordo com o valor de $\mathrm{p}<0,20$ verificado (stepwise). Para verificar a força de associação, foram calculados os coeficientes de correlação de Pearson (r), o coefici- ente de correlação parcial e o coeficiente de determinação.

\section{Resultados}

Entre os diversos grupos de cobertura populacional da ESF avaliados, observamos importantes diferenças quanto à razão de exames citopatológicos nas mulheres entre 25 e 59 anos. No grupo sem ESF, foram realizados em média 17 exames para cada 100 mulheres, enquanto no grupo com mais de $80 \%$ verificou-se que foram realizados em média 26 exames para cada 100 mulheres. A figura 1 demonstra a linearidade da diferença encontrada, indicando que o acesso aos exames preventivos e sua realização são, nos municípios com grande cobertura de Saúde da Família, maior que naqueles sem ou com pequena cobertura.

Encontramos diferenças significativas na realização de CPs entre diferentes grupos de cobertura municipal da

Figura 1. Médias e intervalos de confiança da razão de exames cérvico-vaginais para cada estrato de cobertura municipal de Estratégia Saúde da Família no Rio Grande do Sul, 2006.

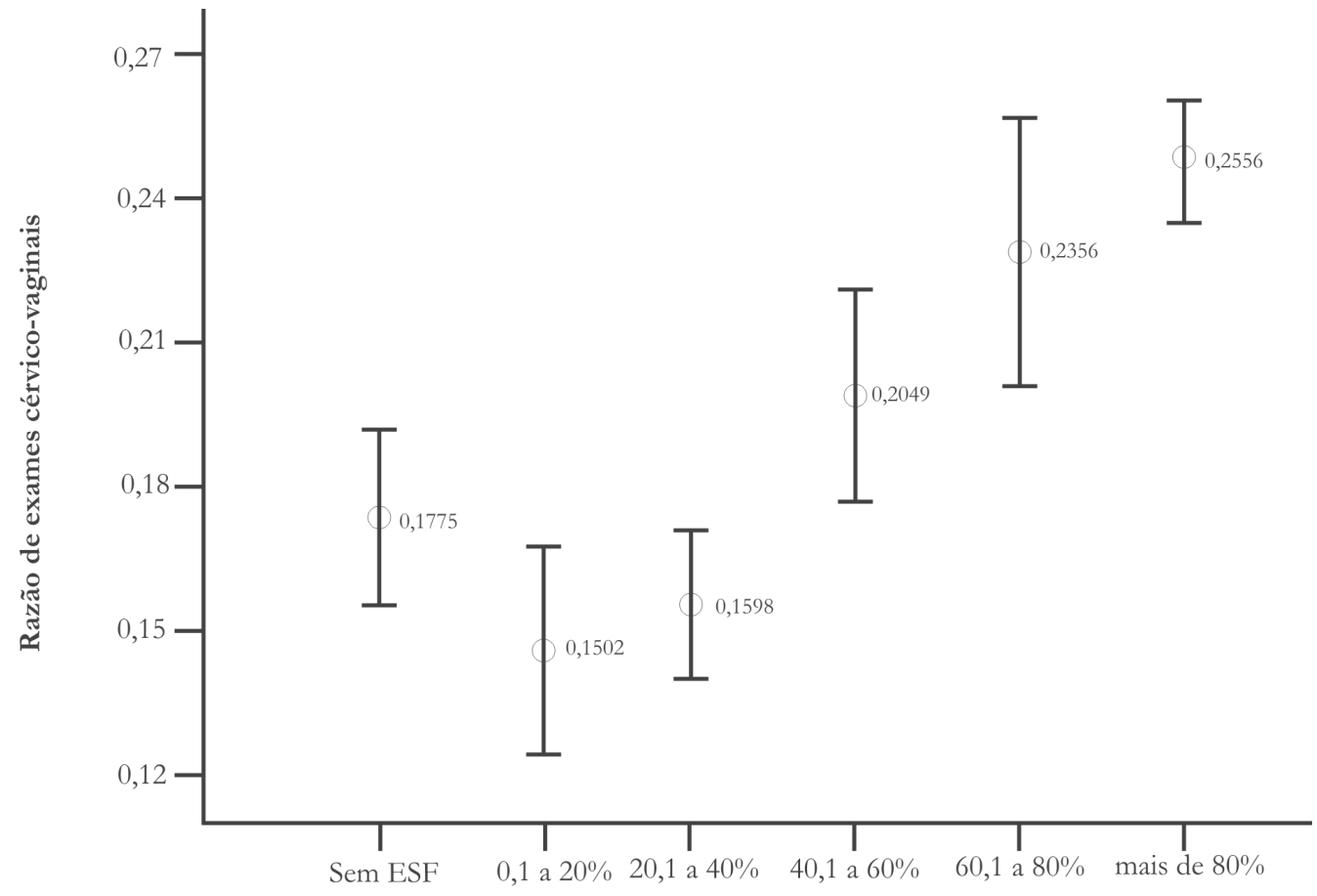

Cobertura Populacional da ESF

123 Rev Bras Med Fam e Com

Rio de Janeiro, v.4, $n^{\circ} 14$, jul /set 2008 
ESF. O valor de F para a análise de variância foi de 20,62 com $\mathrm{p}<0,001$, considerando a totalidade dos municípios (Tabela 1). para cada 100 mulheres. Já entre os municípios com mais de 10 mil, verificou-se que no grupo sem ESF foram

Tabela 1. Comparações das razões de exames citopatológicos entre o grupo de municípios com cobertura populacional da ESF acima de $80 \%$ e os demais grupos de cobertura de ESF, Rio Grande do Sul, 2006.

\begin{tabular}{|c|c|c|c|c|c|}
\hline \multirow{2}{*}{$\begin{array}{l}\text { Grupo de } \\
\text { comparação }\end{array}$} & \multirow{2}{*}{$\begin{array}{c}\text { Grupos de } \\
\text { cobertura ESF }\end{array}$} & \multirow{2}{*}{$\begin{array}{c}\text { Diferenças de médias } \\
\text { de razão de CP } \\
\text { (erro padrão) }\end{array}$} & \multirow{2}{*}{ Valor p } & \multicolumn{2}{|c|}{$95 \%$ Intervalo de confiança } \\
\hline & & & & Inferior & Superior \\
\hline \multirow{5}{*}{$\begin{array}{c}\text { Mais de } 80 \% \text { de } \\
\text { cobertura } \\
\text { populacional } \\
\text { de ESF }\end{array}$} & Sem ESF & $0,078(0,011)^{*}$ & $<0,001$ & 0,0456 & 0,1106 \\
\hline & $0---\mid 20 \% \mathrm{ESF}$ & $0,105(0,015)^{*}$ & $<0,001$ & 0,0622 & 0,1484 \\
\hline & $20--\mid 40 \% \mathrm{ESF}$ & $0,095(0,014)^{*}$ & $<0,001$ & 0,0545 & 0,1363 \\
\hline & $40--\mid 60 \% \mathrm{ESF}$ & $0,050(0,015)^{*}$ & 0,012 & 0,0072 & 0,0934 \\
\hline & $60--\mid 80 \%$ ESF & $0,023(0,015)^{*}$ & 0,649 & $-0,0206$ & 0,0674 \\
\hline
\end{tabular}

Análise de variância - ANOVA (post hoc Tukey)

* Diferença estatisticamente significativa em relação ao grupo de municípios com cobertura de ESF maior de $80 \%$.

grupo de municípios com maior cobertura da ESF (mais de 80\%) apresentou diferença estatisticamente significativa em relação aos municípios com cobertura de ESF até 60\% (grupos 1, 2, 3 e 4 do Quadro 1) com p<0,05. A Tabela 1 demonstra que, nos municípios com mais de $80 \%$ de cobertura, existe uma razão de exames significativamente maior que nos municípios sem ESF, ou com coberturas inferiores a 60\%. Também se comparou o grupo de cobertura de ESF entre 60,01 e 80\% que apresentou diferença estatisticamente significativa com os grupos sem ESF $(p=0,02)$, de 0,01 a $20 \%(p=0,001)$ e de 20,01 a 40\% ( $p=0,003)$.

Destaca-se também que nos municípios com menos de 10 mil habitantes apresentam maiores razões de CP (razão=0,244; desvio padrão=0,103), independentemente da cobertura de ESF, que aqueles municípios com população superior a 10 mil habitantes (razão= 0,158; desvio $=0,610)$, como verificado por meio do teste $T$ entre as médias de razão de CP (teste $\mathrm{T}, \mathrm{F}=38,9$ e $\mathrm{p}<0,0001$ ).

Analisando separadamente os municípios com menos de 10 mil habitantes, observamos que persistem diferenças quanto à razão de exames citopatológicos nos grupos de cobertura populacional da ESF. A Figura 2 mostra que no grupo sem ESF foram realizados em média 20 exames para cada 100 mulheres, enquanto no grupo com mais de $80 \%$ verificou-se que foram realizados em média 26 exames realizadas em média 14 exames, enquanto no grupo com mais de $80 \%$ foram realizados 20 exames para cada 100 mulheres.

Observou-se que a diferença na razão de exames CP permanece estatisticamente significativa para os grupos de cobertura de ESF avaliados, mesmo analisando separadamente os municípios com mais de 10 mil e com menos de 10 mil habitantes (respectivamente, $F=3,89 p<0,002$ e $\mathrm{F}=4,77 \mathrm{p}<0,001)$. Conforme demonstrado na Tabela 2, o grupo de maior cobertura da ESF (municípios com mais de $80 \%$ apresentou diferença significativamente maior na razão de exames CP que os municípios que não aderiram a ESF nos dois agrupamentos (municípios com mais e menos que 10 mil habitantes, $\mathrm{p}<0,01)$. Há uma tendência para a diferença entre os grupos de municípios com 60,01\% a $80 \%$ e o grupo entre $0,01 \%$ a $40 \%$ nos municípios com mais de 10 mil $(p=0,11)$.

Ao realizar-se regressão linear múltipla, encontrouse associação moderada $(r=0,42)$ entre cobertura da ESF e a razão de exames preventivos para o câncer de colo uterino. Segundo este modelo ajustado para o tamanho da população, para cada $10 \%$ a mais de cobertura populacional, a razão de cobertura de CP na população feminina aumenta em 0,01. Além disso, estas duas variáveis explicam cerca de $17 \%$ da variação da razão de exames $\left(R^{2}\right.$ ajustado $\left.=0,176\right)$.

124 Rev Bras Med Fam e Com

Rio de Janeiro, v.4, nº 14, jul /set 2008 
Figura 2. Médias e intervalos de confiança da razão de exames cérvico-vaginais para cada estrato de cobertura municipal de Estratégia Saúde da Família dos municípios com mais de 10 mil e com menos de 10 mil habitantes no Rio Grande do Sul, 2006.
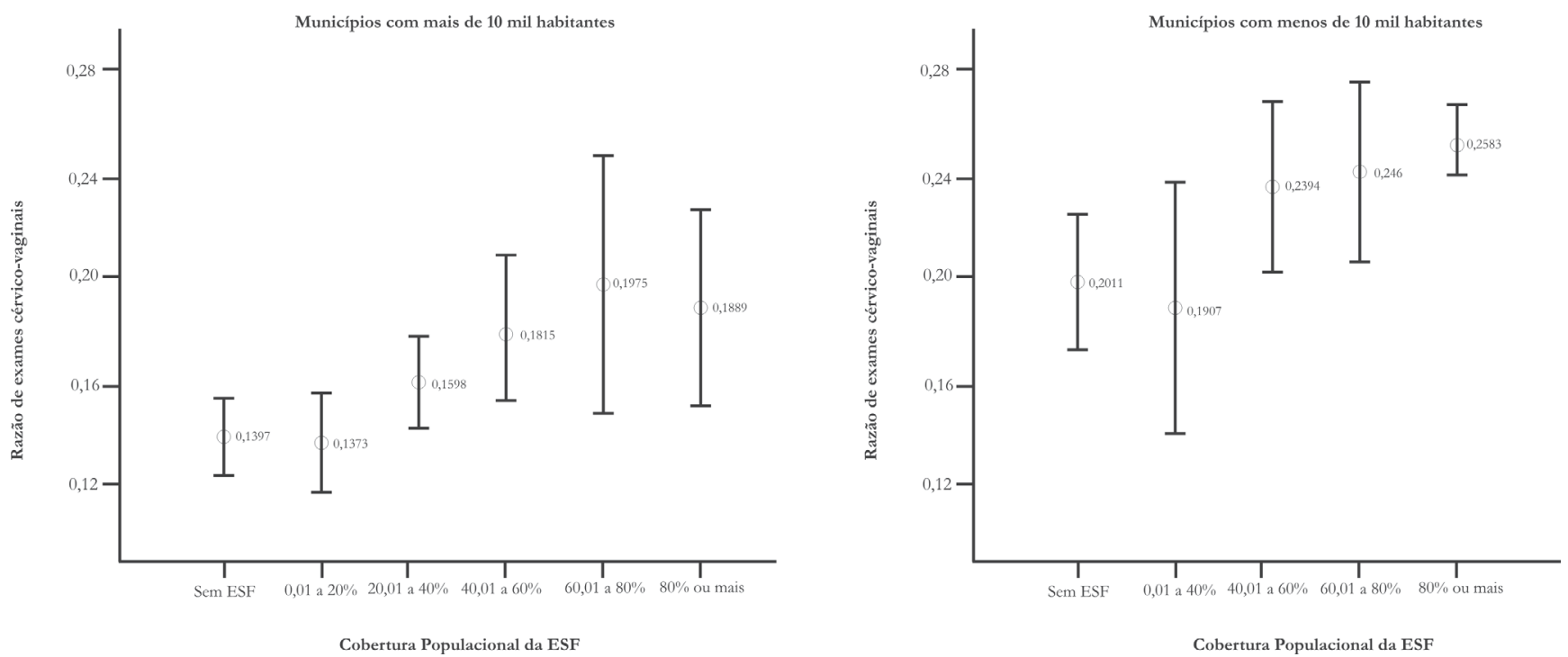

Tabela 2. Comparações das razões de exames citopatológicos entre o grupo de municípios com cobertura populacional da ESF acima de $80 \%$ e os demais grupos de cobertura populacional da ESF para municípios com mais de 10 mil habitantes, Rio Grande do Sul, 2006.

\begin{tabular}{cccccc}
\hline $\begin{array}{c}\text { Grupo de } \\
\text { comparação }\end{array}$ & $\begin{array}{c}\text { Grupos de } \\
\text { cobertura ESF }\end{array}$ & $\begin{array}{c}\text { Diferença de médias } \\
\text { (erro padrão) }\end{array}$ & P valor & \multicolumn{2}{c}{$\mathbf{9 5 \% \text { Intervalo de confiança }}$} \\
\cline { 5 - 6 } & Sem ESF & $0,0572(0,015)^{*}$ & 0,003 & 0,0138 & 0,1007 \\
$\begin{array}{c}\text { Mais de } 80 \% \text { de } \\
\text { cobertura }\end{array}$ & $0--\mid 20 \%$ & $0,0397(0,039)$ & 0,910 & $-0,0715$ & 0,1510 \\
$\begin{array}{c}\text { populacional de ESF } \\
\text { em municípios com } \\
\text { mais de } 10 \text { mil } \\
\text { habitantes }\end{array}$ & $20--\mid 40 \%$ & $0,0954(0,039)$ & 0,139 & $-0,0158$ & 0,2067 \\
$\begin{array}{c}\text { Mais de } 80 \% \text { de } \\
\text { cobertura }\end{array}$ & $40--\mid 60 \%$ & $0,0188(0,025)$ & 0,974 & $-0,0522$ & 0,0900 \\
$\begin{array}{c}\text { populacional de ESF } \\
\text { em municípios com } \\
\text { menos de 10 mil } \\
\text { habitantes }\end{array}$ & $00-\mid 80 \%$ & $0,0123(0,020)$ & 0,989 & $-0,0441$ & 0,0688 \\
\hline
\end{tabular}

* Diferença estatisticamente significativa em relação ao grupo de municípios com cobertura de ESF maior de $80 \%$.

\# O grupo de municípios com cobertura entre $0,01 \%$ a $20 \%$ e entre $20,01 \%$ e $40 \%$ foram agrupados por apresentarem pequeno número em cada estrato.

\section{Discussão}

Nossos resultados mostram que o modelo de Atenção Primária à Saúde pode representar um importante preditor de realização de CP. Municípios com maior cobertura da ESF no RS têm a razão de realização de CPs na população de interesse significativamente maior. Também é impor- tante ressaltar que essa diferença persiste quando analisamos somente os municípios com mais de 10 mil habitantes, onde a ESF encontrou maior dificuldade para ser implementada, mesmo com coberturas menores.

A ESF apresenta um conjunto de características da atenção e do cuidado como maior acesso, integralidade 
e competência cultural ${ }^{8}$ para atuar justamente sobre os agravos mais prevalentes, desenvolvendo ações preventivas de rotina como o rastreamento com CP para o câncer de colo. É importante salientar que parte do sucesso da rotina de realização deste exame pode ser atribuído à relação profissional-paciente ${ }^{18}$, ou seja, nos municípios com mais equipes de Saúde da Família uma maior parcela da população reconhece seu médico e equipe como referências que conhecem sua história de vida e de saúde (longitudinalidade), e, por sua vez, reconhecem pacientes faltosos, sob sua responsabilidade.

Além de gerar hipóteses, o estudo ecológico tem importante função no planejamento de políticas públicas em saúde porque permite uma avaliação global da população. No processo de pactuação entre a gestão municipal, estadual e federal do indicador razão de exames citopatológicos em mulheres entre 25 e 59 anos, é necessário considerar a dinâmica de implantação da Estratégia Saúde da Família e sua cobertura. No presente trabalho, para cada aumento de $10 \%$ na cobertura populacional da ESF, ocorre o aumento de 0,01 na razão de CP. Ou seja, um município com menos de 10 mil sem ESF, que apresenta em média uma razão de 0,201, de acordo com o observado no RS em 2006, se tivesse $100 \%$ de cobertura da população por ESF, teria uma razão de aproximadamente 0,301, alcançando o valor de referência no SISPACTO.

Ao descrever as associações e comparações entre grupos é imprescindível ponderar sobre as limitações dos resultados encontrados. A análise ecológica de nosso estudo apresenta duas limitações importantes. A primeira está relacionada ao viés metodológico por si, uma vez que não podemos garantir que as populações adscritas à ESF sejam, dentro de cada município, as que mais realizam CP. O rastreamento poderia estar fundamentalmente concentrado nas áreas não cobertas. Além disso, o indicador de razão de exames não permite distinguir situações em que um pequeno grupo de mulheres com mais facilidade de acesso ao serviço realizam o CP com intervalos mais curtos que os recomendados, ou por apresentarem alterações no resultado do mesmo, o que indicaria o aumento da frequen- cia do exame. No entanto, a linearidade observada ao longo dos estratos de cobertura progressivamente maior de ESF e a inclusão de municípios com 100\% de cobertura diminuem a probabilidade desse viés.

A segunda limitação importante é o fato de não ter sido feito o controle para fatores já estabelecidos na literatura, como renda, escolaridade e distribuição etária da população - por exemplo, dentro da faixa etária estudada, uma maior densidade de mulheres com menos de 35 anos em um município poderia influenciar negativamente a razão de exames. Essa limitação levanta a necessidade de futura correção dos resultados pelos confundidores descritos

Nosso estudo responde em parte ao questionamento levantado na revisão sistemática de Martins ${ }^{17}$ quanto à importância de preditores da cobertura de CP relacionados à questão da organização do serviço de Atenção Primária à Saúde. O modelo da ESF, que repetidamente vem mostrando-se superior às unidades básicas de saúde tradicionais nos estudos comparativos, parece ser um preditor da cobertura populacional do rastreamento do câncer de colo uterino.

Por outro lado, parece ser importante avaliar com outra metodologia a experiência dos municípios com menos de 10 mil habitantes que, apesar de não terem aderido à ESF (cobertura populacional igual a zero) apresentam razão de exames intermediária entre a baixa e alta cobertura. O acompanhamento controlado pelo tempo de implantação da ESF em altas coberturas nos municípios também permitirá, em breve, análises quanto à associação com mortalidade pelo câncer de colo uterino. No entanto, esses resultados preliminares justificam o esforço por uma maior cobertura da ESF, independentemente do porte populacional do município.

\section{Conclusões}

Diante de nossos resultados, a organização do modelo de APS mostra-se como um fator associado à melhora de processos de atenção à saúde da mulher, a exemplo do que já vem sendo demonstrado para a saúde infantil. A análise comparada por crescentes padrões de cobertura da

126 Rev Bras Med Fam e Com

Rio de Janeiro, v.4, $n^{\circ} 14$, jul /set 2008 
ESF reforça o planejamento do SUS em relação ao caráter substitutivo dessa estratégia sobre o modelo da Atenção Básica tradicional, com metas de cobertura crescente da população, ultrapassando $80 \%$.

\section{Referências}

1. Macinko J, Starfield B, Shi L. The contribution of primary care systems to health outcomes within organization for economic cooperation and development (OECD) countries, 1970-1998. Health Erv Res. 2003;38:831- 65. 2. Starfield B, Shi L, Macinko J. Contribution of primary care to health systems and health. Milbank Q. 2005;83:457-502. 3. Starfield B. Atenção Pimária: equilíbrio entre necessidades de saúde, serviços e tecnologia. Brasília (DF): UNESCO Brasil, Ministério da Saúde; 2004. p.43-70.

4. Brasil.Ministério da Saúde. Departamento de Atenção Básica. Evolução e série histórica de implantação da ESF: relatório de busca. Brasília (DF): DAB; 2008.

5. Macinko J, Guanais FC, Souza MFM. Evaluation of the impact of the Family Health Program on infant mortality in Brazil, 1990-2002. J Epidemiol Community Health. 2006; 60: 13-19.

6. Harzheim E. Evaluación de la atención a la salud infantil del Programa Saúde da Família en la región sur de Porto Alegre, Brasil. [Tese de doutorado]. Alicante, Espanha, Departamento de Salud Pública, Universidad de Alicante; 2004

7. Harzheim E, Starfield B, Rajmil L, Dardet CA, Stein AT. Consistência interna e confiabilidade da versão em português do Instrumento de Avaliação da Atenção Primária (PCATool-Brasil) para serviços de saúde infantil. Cad. Saúde Pública. ago 2006; 22 (8): 1649-1659.

8. Oliveira MMC. Presença e extensão dos atributos da Atenção Primária à Saúde entre os serviços de Atenção Primária em Porto Alegre: uma análise agregada. [Dissertação de Mestrado] Porto Alegre, Programa de PósGraduação em Epidemiologia, Faculdade de Medicina, Universidade Federal do Rio Grande do Sul; 2007.

9. Macinko J, Almeida C, Sá PK. A rapid assessment methodology for the evaluation of primary care organization and performance in Brazil. Helath Policy and
Planning 2007; 22: 167-177.

10. Brasil. Ministério da Saúde: portaria Gabinete Ministerial no 325. Brasília, DF. 21 de fevereiro de 2008.

11. Brasil. Ministério da Saúde. Instituto Nacional de Câncer. Incidência de câncer de colo de útero no Brasil por unidades federativas, Brasil: 1997-1998. Rio de Janeiro: INCA; 2008. (Cadernos de Atenção Básica; 13).

12. Brasil. Ministério da Saúde. Departamento de Informação e Informática do SUS. Sistema de informação sobre mortalidade 2003: dados de declaração de óbito. Brasília (DF): DATASUS; 2008.

13. International Agency for Research on Cancer (IARC) Working Group on the Evaluation of Cervical Cancer Screening Programmes. Screening for squamous cervical cancer: duration of low risk after negative results of cervical cytology and its implication for screening policies. Br Med J 1986; 293(6548):659-664.

14. Sasieni PD, Cuzick J, Lynch-Farmery E. Estimating the efficacy of screening by auditing smear histories of women with and without cervical cancer. The National Coordinating Network for Cervical Screening Working Group. Br J Cancer. 1996;73(8):1001-1005.

15. Sawaya GF, Kerlikowske K, Lee NC, Gildengorin G, Washington AE. Frequency of cervical smear abnormalities within 3 years of normal cytology. Obstet Gynecol. 2000; 96(2):219-23.

16. Brasil. Ministério da Saúde. Secretaria de Atenção à Saúde. Departamento de Atenção Básica. Controle dos Cânceres do Colo do Útero e da Mama. Brasília (DF): Secretaria de Atenção à Saúde; 2006. (Série A: Normas e Manuais Técnicos). Disponível em http://dtr2004.saude. gov.br/dab/docs/publicacoes/cadernosab/abcad13.pdf Acesso em 10 de abril de 2008.

17. Martins LFL, Thuler LCS, Valente JG. Cobertura do exame de Papanicolaou no Brasil e seus fatores determinantes: uma revisão sistemática da literatura. Rev Bras Ginecol Obstet. 2005; 27: 485-92.

18.Oliveira MMHN, Silva AAM, Brito LMO, Coimbra LC. Cobertura e fatores associados à não realização do exame preventivo de Papanicolaou em São Luís, Maranhão. Rev 
Bras Epidemiol. 2006; 9(3): 325-34.

\section{Endereço para correspondência:}

Rua Ramiro Barcelos 2400, térreo.

Porto Alegre, RS.

CEP: 90035-003

\section{Endereço eletrônico:}

paulofontanive@gmail.com 